\title{
Quantum entanglement and teleportation using statistical correlations ${ }^{\dagger}$
}

\author{
ATUL KUMAR and MANGALA SUNDER KRISHNAN* \\ Department of Chemistry, Indian Institute of Technology Madras, Chennai 600036 \\ e-mail: mangal@iitm.ac.in
}

\begin{abstract}
A study of quantum teleportation using two and three-particle correlated density matrix is presented. A criterion based on standard quantum statistical correlations employed in the many-body virial expansion is used to determine the extent of entanglement for a $2 \mathrm{~N}$-particle system. A relation between the probability and statistical parameters is established using the correlated density matrices for the particles.
\end{abstract}

Keywords. Entanglement; quantum correlation; teleportation; density matrix; quantum information.

\section{Introduction}

Quantum entanglement plays a vital role in many communication protocols such as teleportation, dense coding, entanglement swapping and data compression. ${ }^{1-4}$ It rests on quantum correlations for which there are no classical analogues. It is important to understand and characterize entanglement in different systems, e.g. Bennett et al have shown the teleportation of a single particle using the celebrated Bohm-Aharanov-Einstein-Podolsky-Rosen (EPR) pair as a quantum channel. ${ }^{5,6}$ For two-particle (bipartite) systems there are several measures to quantify the entanglement and quantum entanglement is well-understood, however, generalization of the same to study and quantify entanglement in many-particle systems is still an open problem. ${ }^{7-11}$ Experimental studies on the creation of a few entangled states have been reported recently. ${ }^{12-16}$

In this article, we propose quantum statistical correlations as a measure of entanglement for multiparticle systems which is given in section 2 . The criterion to define the extent of correlation will be developed in a step-wise process by considering some standard examples of entangled systems. In addition, a simple generalization to produce a $2 \mathrm{~N}$ particle entangled channel starting from a fourparticle entangled state is described. In section 3 we review some of the teleportation schemes using density operators and establish a relation between statistical parameters and probability of teleportation process.

\footnotetext{
${ }^{\dagger}$ Dedicated to the memory of the late Professor S K Rangarajan

*For correspondence
}

\section{Multi-particle entanglement}

In this section, the entanglement properties of twoparticle entangled states and generalization of the same to multi-particle systems are discussed. We assess the extent of entanglement based on wellestablished statistical mechanical formula for correlation coefficients. ${ }^{17-19}$ The approach presented here applies to statistical ensembles, and therefore to electrons and other spin-1/2 systems as well as photons. Interactions between them do include correlations of the type described here. A detailed justification for the use of a simplified form of correlated density matrix is given by Fano. ${ }^{20}$ The use of correlation measures of multi-particle systems through Ursell-Mayer type cluster coefficients for determining correlations between spins (particles) is suggested as a means for removing ambiguities in defining the degree of entanglement between multiple particles. Ursell-Mayer type correlated density matrices for multi-particles are extensions to the two-particle density operator used here. There are standard procedures for determining statistical correlations between particles in an $N$-particle density matrix which will be considered in the future for theoretical description of quantum information processing. ${ }^{21,22}$

\subsection{Two and three-particle systems}

Correlation coefficients for two spin-1/2 (two qubit) systems are defined as

$$
C_{i j}^{12}=\left\langle\sigma_{i}^{1} \sigma_{j}^{2}\right\rangle-\left\langle\sigma_{i}^{1}\right\rangle\left\langle\sigma_{j}^{2}\right\rangle
$$


where $\sigma^{\prime}$ s are Pauli spin matrices $\{i, j=x, y, z\}$, and the coefficients $C_{i j}^{12}$ are components of a second rank symmetric traceless tensor $\underset{\tilde{s}}{\boldsymbol{C}}$. The averages are calculated for the four Bell states, namely

$$
|\psi\rangle_{12}^{ \pm}=\frac{|01\rangle_{12} \pm|10\rangle_{12}}{\sqrt{2}} \text { and }|\phi\rangle_{12}^{ \pm}=\frac{|00\rangle_{12} \pm|11\rangle_{12}}{\sqrt{2}}
$$

and the non-zero correlation coefficients are listed in table 1 . The maximum value $( \pm 1)$ of non-zero correlation coefficients indicates that the states are maximally entangled. For product states such as $|0\rangle_{1} \otimes\left\{|0\rangle_{2}+|1\rangle_{2}\right\}$ all the correlation coefficients are zero. However, the maximum value of a single correlation coefficient alone does not ensure that a given system is maximally entangled, e.g. a system whose density operator is $\rho^{12}=1 / 2\left[|00\rangle_{12}\left\langle\left. 00\right|_{12}\right.\right.$ $+|11\rangle_{12}\left\langle\left. 11\right|_{12}\right]$, where two-particles are in a mixed state, shows $C_{z z}^{12}=1$. The two-particle system represented here is not entangled, though correlated in a measurement sense. Thus to ensure maximum entanglement more than one information is needed i.e. either more than one data should be available with respect to non-zero correlation-coefficients or the state in question must be pure along with a nonzero correlation coefficient. The fact that the four Bell-states are pure and possess more than one nonzero correlation coefficients shows that the correlations between the particles are quantum. ${ }^{23}$ Correlation coefficients for three-particle systems are defined as

$$
\begin{aligned}
C_{i j k}^{123}=\left\langle\sigma_{i}^{1} \sigma_{j}^{2} \sigma_{k}^{3}\right\rangle & -\left\langle\sigma_{i}^{1}\right\rangle\left\langle\sigma_{j}^{2} \sigma_{k}^{3}\right\rangle-\left\langle\sigma_{j}^{2}\right\rangle\left\langle\sigma_{i}^{1} \sigma_{k}^{3}\right\rangle \\
& -\left\langle\sigma_{k}^{3}\right\rangle\left\langle\sigma_{i}^{1} \sigma_{j}^{2}\right\rangle+2\left\langle\sigma_{i}^{1}\right\rangle\left\langle\sigma_{j}^{2}\right\rangle\left\langle\sigma_{k}^{3}\right\rangle,
\end{aligned}
$$

where $C_{i j k}^{123}$ are components of a third rank tensor. The averages are calculated for three-particle Greenberger-Horne-Zeilinger ${ }^{24}$ (GHZ) states and a set of states such as

Table 1. Non-zero correlation coefficients associated with four Bell states.

\begin{tabular}{ccccc}
\hline & $|\psi\rangle_{12}^{-}$ & $|\psi\rangle_{12}^{+}$ & $|\phi\rangle_{12}^{-}$ & $|\phi\rangle_{12}^{+}$ \\
\hline$C_{x x}^{12}$ & -1 & +1 & -1 & +1 \\
$C_{y y}^{12}$ & -1 & +1 & +1 & -1 \\
$C_{z z}^{12}$ & -1 & -1 & +1 & +1 \\
\hline
\end{tabular}

$$
\begin{aligned}
& |\chi\rangle_{123}^{(1),(2)}=\frac{1}{\sqrt{2}}\left[|\phi\rangle_{13}^{+}|0\rangle_{2} \pm|\phi\rangle_{13}^{-}|1\rangle_{2}\right], \\
& |\chi\rangle_{123}^{(3),(4)}=\frac{1}{\sqrt{2}}\left[|\phi\rangle_{13}^{+}|1\rangle_{2} \pm|\phi\rangle_{13}^{-}|0\rangle_{2}\right] \\
& |\chi\rangle_{123}^{(5),(6)}=\frac{1}{\sqrt{2}}\left[|\psi\rangle_{13}^{+}|0\rangle_{2} \pm|\psi\rangle_{13}^{-}|1\rangle_{2}\right] \\
& |\chi\rangle_{123}^{(7),(8)}=\frac{1}{\sqrt{2}}\left[|\psi\rangle_{13}^{+}|1\rangle_{2} \pm|\psi\rangle_{13}^{-}|0\rangle_{2}\right]
\end{aligned}
$$

and the corresponding non-zero correlation coefficients are listed in table 2. The set of GHZ states and the states represented by (4) possess the same degree of correlation between the three-particles, as the set represented by (4) can be obtained through three-particle GHZ states by just doing local operations on particle 2 . The main difference between the two sets lies in the robustness shown by the set of states represented by (4) with respect to second particle such that even if second particle is traced out, $|\chi\rangle_{123}^{(i)}$ can still be used as a quantum channel and is useful in eliminating any intermediate observer controlling or monitoring the teleportation process. However, in the case of GHZ states if any one of the particle is traced out then the remaining system does not possess quantum correlations. Interestingly, the non-zero correlation coefficients for a three-particle W state $^{25}$

$$
|\psi\rangle_{123}^{W}=\frac{1}{\sqrt{3}}\left[|010\rangle_{123}+|001\rangle_{123}+|100\rangle_{123}\right]
$$

show the value $(\sim 0.45)$ which is less than maximum indicating that the correlation between the particles is not maximal.

\subsection{Four-particle systems and generalization}

The correlation tensor for a four spin- $1 / 2$ system is given by the coefficients $C_{i j k l}^{1234}\{i, j, k, l=x, y, z\}$ where

$$
\begin{aligned}
C_{i j k l}^{123}= & \left\langle\sigma_{i}^{1} \sigma_{j}^{2} \sigma_{k}^{3} \sigma_{l}^{4}\right\rangle-\left\langle\sigma_{i}^{1}\right\rangle\left\langle\sigma_{j}^{2} \sigma_{k}^{3} \sigma_{l}^{4}\right\rangle \\
& -\left\langle\sigma_{j}^{2}\right\rangle\left\langle\sigma_{i}^{1} \sigma_{k}^{3} \sigma_{l}^{4}\right\rangle-\left\langle\sigma_{k}^{3}\right\rangle\left\langle\sigma_{i}^{1} \sigma_{j}^{2} \sigma_{l}^{4}\right\rangle \\
& -\left\langle\sigma_{l}^{4}\right\rangle\left\langle\sigma_{i}^{1} \sigma_{j}^{2} \sigma_{k}^{3}\right\rangle+2\left\langle\sigma_{i}^{1}\right\rangle\left\langle\sigma_{j}^{2}\right\rangle\left\langle\sigma_{k}^{3} \sigma_{l}^{4}\right\rangle \\
& +2\left\langle\sigma_{i}^{1}\right\rangle\left\langle\sigma_{k}^{3}\right\rangle\left\langle\sigma_{j}^{2} \sigma_{l}^{4}\right\rangle+2\left\langle\sigma_{i}^{1}\right\rangle\left\langle\sigma_{l}^{4}\right\rangle\left\langle\sigma_{j}^{2} \sigma_{k}^{3}\right\rangle
\end{aligned}
$$


Table 2. Non-zero correlation coefficients associated with three-particle GHZ states and three-particle states represented by $|\chi\rangle_{123}^{(i)}$ (4).

\begin{tabular}{lcccccc}
\hline & $C_{x x x}^{123}$ & $C_{y y x}^{123}$ & $C_{y x y}^{123}$ & $C_{x y y}^{123}$ & $C_{x z x}^{123}$ & $C_{y z y}^{123}$ \\
\hline$\frac{1}{\sqrt{2}}[000 \pm 111]$ & \pm 1 & $\mp 1$ & $\mp 1$ & $\mp 1$ & - & - \\
$\frac{1}{\sqrt{2}}[001 \pm 110]$ & \pm 1 & $\mp 1$ & \pm 1 & \pm 1 & - & - \\
$\frac{1}{\sqrt{2}}[010 \pm 101]$ & \pm 1 & \pm 1 & $\mp 1$ & \pm 1 & - & - \\
$\frac{1}{\sqrt{2}}[011 \pm 100]$ & \pm 1 & \pm 1 & \pm 1 & $\mp 1$ & - & - \\
$|\chi\rangle_{123}^{(1)}$ & - & -1 & - & -1 & - & +1 \\
$|\chi\rangle_{123}^{(2)}$ & - & -1 & - & -1 & -1 & -1 \\
$|\chi\rangle_{123}^{(3)}$ & - & +1 & - & +1 & +1 & -1 \\
$|\chi\rangle_{123}^{(4)}$ & - & +1 & - & +1 & -1 & +1 \\
$|\chi\rangle_{123}^{(5)}$ & - & +1 & - & -1 & +1 & +1 \\
$|\chi\rangle_{123}^{(6)}$ & - & +1 & - & -1 & -1 & -1 \\
$|\chi\rangle_{123}^{(7)}$ & - & -1 & - & +1 & -1 & -1 \\
$|\chi\rangle_{123}^{(8)}$ & - & -1 & - & +1 & +1 & +1 \\
\hline
\end{tabular}

$$
\begin{aligned}
& +2\left\langle\sigma_{j}^{2}\right\rangle\left\langle\sigma_{k}^{3}\right\rangle\left\langle\sigma_{i}^{1} \sigma_{l}^{4}\right\rangle+2\left\langle\sigma_{j}^{2}\right\rangle\left\langle\sigma_{l}^{4}\right\rangle\left\langle\sigma_{i}^{1} \sigma_{k}^{3}\right\rangle \\
& +2\left\langle\sigma_{k}^{3}\right\rangle\left\langle\sigma_{l}^{4}\right\rangle\left\langle\sigma_{i}^{1} \sigma_{j}^{2}\right\rangle-\left\langle\sigma_{i}^{1} \sigma_{j}^{2}\right\rangle\left\langle\sigma_{k}^{3} \sigma_{l}^{4}\right\rangle \\
& -\left\langle\sigma_{i}^{1} \sigma_{k}^{3}\right\rangle\left\langle\sigma_{j}^{2} \sigma_{l}^{4}\right\rangle-\left\langle\sigma_{i}^{1} \sigma_{l}^{4}\right\rangle\left\langle\sigma_{j}^{2} \sigma_{k}^{3}\right\rangle \\
& -6\left\langle\sigma_{i}^{1}\right\rangle\left\langle\sigma_{j}^{2}\right\rangle\left\langle\sigma_{k}^{3}\right\rangle\left\langle\sigma_{l}^{4}\right\rangle .
\end{aligned}
$$

The general expression for the $N$-particle correlation coefficients can be obtained by solving the equations for quantum cluster functions derived formally from the $N$-th quantum virial coefficient (quantum trace).

The non-zero correlation coefficients corresponding to maximally entangled four-particle GHZ states show maximum value and are listed in table 3 . The generalized four-particle $\mathrm{W}$ state

$$
\begin{aligned}
|\psi\rangle_{1234}^{W}= & \frac{1}{2}\left[|0001\rangle_{1234}+|0010\rangle_{1234}\right. \\
& \left.+|0100\rangle_{1234}+|1000\rangle_{1234}\right]
\end{aligned}
$$

shows a value of $(\sim 0.25)$, for correlation coefficients, which suggests that the state in question is less than maximally entangled. Rigolin ${ }^{26}$ proposed a set of four-particle generalized Bell basis to teleport an arbitrary two-particle state, however, all the correlation coefficients for the set show zero value which suggests that there is no genuine correlations between the four-particles. Yeo and $\mathrm{Chua}^{27}$ proposed a genuinely entangled four-particle state to accomplish the same task and the non-zero correlation coefficients corresponding to the set proposed are listed in table 4 . The set of four-particle cluster states, ${ }^{16,28}$ namely

$$
\begin{aligned}
& |\chi\rangle_{1234}^{(1)-(16)}=\frac{1}{\sqrt{2}}\left[\left(\begin{array}{l}
|0\rangle \\
|1\rangle
\end{array}\right)_{1} \otimes\left(\begin{array}{l}
|\phi\rangle^{+} \\
|\psi\rangle^{+}
\end{array}\right)_{23} \otimes\left(\begin{array}{l}
|0\rangle \\
|1\rangle
\end{array}\right)_{4}\right. \\
& \left. \pm\left(\begin{array}{l}
|1\rangle \\
|0\rangle
\end{array}\right)_{1} \otimes\left(\begin{array}{l}
|\phi\rangle^{-} \\
|\psi\rangle^{-}
\end{array}\right)_{23} \otimes\left(\begin{array}{l}
|1\rangle \\
|0\rangle
\end{array}\right)_{4}\right]
\end{aligned}
$$

possess genuine correlations between particles and the non-zero correlation coefficients are listed in table 5. Thus for maximally entangled states correlation coefficients show maximum value, for product states they show zero values and for states which are 
Table 3. Non-zero correlation coefficients associated with four-particle GHZ states.

\begin{tabular}{llllllllll}
\hline & $C_{x x x x}^{1234}$ & $C_{x x y y}^{1234}$ & $C_{x y x y}^{1234}$ & $C_{x y y x}^{1234}$ & $C_{y x x y}^{1234}$ & $C_{y x y x}^{1234}$ & $C_{y y x x}^{1234}$ & $C_{y y y y}^{1234}$ & $C_{z z z z}^{1234}$ \\
\hline$\frac{1}{\sqrt{2}}[0000 \pm 1111]$ & \pm 1 & $\mp 1$ & $\mp 1$ & $\mp 1$ & $\mp 1$ & $\mp 1$ & $\mp 1$ & \pm 1 & -2 \\
$\frac{1}{\sqrt{2}}[0001 \pm 1110]$ & \pm 1 & \pm 1 & \pm 1 & $\mp 1$ & \pm 1 & $\mp 1$ & $\mp 1$ & $\mp 1$ & +2 \\
$\frac{1}{\sqrt{2}}[0010 \pm 1101]$ & \pm 1 & \pm 1 & $\mp 1$ & \pm 1 & $\mp 1$ & \pm 1 & $\mp 1$ & $\mp 1$ & +2 \\
$\frac{1}{\sqrt{2}}[0011 \pm 1100]$ & \pm 1 & $\mp 1$ & \pm 1 & \pm 1 & \pm 1 & \pm 1 & $\mp 1$ & \pm 1 & -2 \\
$\frac{1}{\sqrt{2}}[0100 \pm 1011]$ & \pm 1 & $\mp 1$ & \pm 1 & \pm 1 & $\mp 1$ & $\mp 1$ & \pm 1 & $\mp 1$ & +2 \\
$\frac{1}{\sqrt{2}}[0101 \pm 1010]$ & \pm 1 & \pm 1 & $\mp 1$ & \pm 1 & \pm 1 & $\mp 1$ & \pm 1 & \pm 1 & -2 \\
$\frac{1}{\sqrt{2}}[0110 \pm 1001]$ & \pm 1 & \pm 1 & \pm 1 & $\mp 1$ & $\mp 1$ & \pm 1 & \pm 1 & \pm 1 & -2 \\
$\frac{1}{\sqrt{2}}[0111 \pm 1000]$ & \pm 1 & $\mp 1$ & $\mp 1$ & $\mp 1$ & \pm 1 & \pm 1 & \pm 1 & $\mp 1$ & +2 \\
\hline
\end{tabular}

Table 4. Non-zero correlation coefficients associated with 16 four-particle states proposed by Yeo and Chua (ref 27).

\begin{tabular}{|c|c|c|c|c|}
\hline & $C_{x y y x}^{1234}$ & $C_{x z z x}^{1234}$ & $C_{z y y z}^{1234}$ & $C_{z z z z}^{1234}$ \\
\hline$|\chi\rangle_{A_{3} A_{4} B_{3} B_{4}}^{(00)}$ & -1 & +1 & -1 & +1 \\
\hline$I^{1} \otimes \sigma_{x}^{2}|\chi\rangle_{A_{3} A_{4} B_{3} B_{4}}^{(00)}$ & +1 & -1 & +1 & -1 \\
\hline$I^{1} \otimes \sigma_{y}^{2}|\chi\rangle_{A_{3} A_{4} B_{3} B_{4}}^{(00)}$ & -1 & -1 & -1 & -1 \\
\hline$I^{1} \otimes \sigma_{z}^{2}|\chi\rangle_{A_{3} A_{4} B_{3} B_{4}}^{(00)}$ & +1 & +1 & +1 & +1 \\
\hline$\sigma_{x}^{1} \otimes I^{2}|\chi\rangle_{A_{3} A_{4} B_{3} B_{4}}^{(00)}$ & -1 & +1 & +1 & -1 \\
\hline$\sigma_{x}^{1} \otimes \sigma_{x}^{2}|\chi\rangle_{A_{3} A_{4} B_{3} B_{4}}^{(00)}$ & +1 & -1 & -1 & +1 \\
\hline$\sigma_{x}^{1} \otimes \sigma_{y}^{2}|\chi\rangle_{A_{3} A_{4} B_{3} B_{4}}^{(00)}$ & -1 & -1 & +1 & +1 \\
\hline$\sigma_{x}^{1} \otimes \sigma_{z}^{2}|\chi\rangle_{A_{3} A_{4} B_{3} B_{4}}^{(00)}$ & +1 & +1 & -1 & -1 \\
\hline$\sigma_{y}^{1} \otimes I^{2}|\chi\rangle_{A_{3} A_{4} B_{1} B_{2}}^{(0)}$ & +1 & -1 & +1 & -1 \\
\hline$\sigma_{y}^{1} \otimes \sigma_{x}^{2}|\chi\rangle_{A_{3} A_{4} B_{1} B_{2}}^{(00)}$ & -1 & +1 & -1 & +1 \\
\hline$\sigma_{y}^{1} \otimes \sigma_{y}^{2}|\chi\rangle_{A_{3} A_{4} B_{1} B_{2}}^{(00)}$ & +1 & +1 & +1 & +1 \\
\hline$\sigma_{y}^{1} \otimes \sigma_{z}^{2}|\chi\rangle_{A_{3} A_{4} B_{1} B_{2}}^{(00)}$ & -1 & -1 & -1 & -1 \\
\hline$\sigma_{z}^{1} \otimes I^{2}|\chi\rangle_{A_{3} A_{4} B_{1} B_{2}}^{(00)}$ & +1 & -1 & -1 & +1 \\
\hline$\sigma_{z}^{1} \otimes \sigma_{x}^{2}|\chi\rangle_{A_{3} A_{4} B_{1} B_{2}}^{(00)}$ & -1 & +1 & +1 & -1 \\
\hline$\sigma_{z}^{1} \otimes \sigma_{y}^{2}|\chi\rangle_{A_{3} A_{4} B_{1} B_{2}}^{(00)}$ & +1 & +1 & -1 & -1 \\
\hline$\sigma_{z}^{1} \otimes \sigma_{z}^{2}|\chi\rangle_{A_{3} A_{4} B_{1} B_{2}}^{(00)}$ & -1 & -1 & +1 & +1 \\
\hline
\end{tabular}


less than maximally entangled their values are less than the maximum.

In addition, it is important to demonstrate a simple way to generalize the four-particle cluster states for the $2 \mathrm{~N}$-particle system using the basis set of (6). The sequential manner in which the $2 \mathrm{~N}$-particle systems are constructed ensures that entanglement properties of these states are preserved down to a pair of particles when they are systematically averaged. Thus a set of six-particle states using (6) is given by

$$
|\chi\rangle_{123456}^{(1)-(64)}=\frac{1}{\sqrt{2}}\left(\left(\begin{array}{l}
|0\rangle \\
|1\rangle
\end{array}\right)_{1}\left(\begin{array}{l}
|\chi\rangle_{2345}^{(1)} \\
|\chi\rangle_{2345}^{(3)} \\
|\chi\rangle_{2345}^{(5)} \\
|\chi\rangle_{2345}^{(7)} \\
|\chi\rangle_{2345}^{(9)} \\
|\chi\rangle_{2345}^{(1)} \\
|\chi\rangle_{2345}^{(13)} \\
|\chi\rangle_{2345}^{(15)}
\end{array}\right) \otimes\left(\begin{array}{l}
|0\rangle \\
|1\rangle
\end{array}\right) 6\right.
$$

Table 5. Non-zero correlation coefficients associated with 16 four-particle cluster states (6).

\begin{tabular}{lcccc}
\hline & $C_{x x y y}^{1234}$ & $C_{x y x y}^{1234}$ & $C_{y x y x}^{1234}$ & $C_{y y x x}^{1234}$ \\
\hline$|\chi\rangle_{1234}^{(1)}$ & +1 & +1 & +1 & +1 \\
$|\chi\rangle_{1234}^{(2)}$ & -1 & -1 & -1 & -1 \\
$|\chi\rangle_{1234}^{(3)}$ & -1 & -1 & +1 & +1 \\
$|\chi\rangle_{1234}^{(4)}$ & +1 & +1 & -1 & -1 \\
$|\chi\rangle_{1234}^{(5)}$ & -1 & +1 & -1 & +1 \\
$|\chi\rangle_{1234}^{(6)}$ & +1 & -1 & +1 & -1 \\
$|\chi\rangle_{1234}^{(7)}$ & +1 & -1 & -1 & +1 \\
$|\chi\rangle_{1234}^{(8)}$ & -1 & +1 & +1 & -1 \\
$|\chi\rangle_{1234}^{(9)}$ & +1 & +1 & -1 & -1 \\
$|\chi\rangle_{1234}^{(10)}$ & -1 & -1 & +1 & +1 \\
$|\chi\rangle_{1234}^{(11)}$ & -1 & -1 & -1 & -1 \\
$|\chi\rangle_{1234}^{(12)}$ & +1 & +1 & +1 & +1 \\
$|\chi\rangle_{1234}^{(13)}$ & -1 & +1 & +1 & -1 \\
$|\chi\rangle_{1234}^{(14)}$ & +1 & -1 & -1 & +1 \\
$|\chi\rangle_{1234}^{(15)}$ & +1 & -1 & +1 & -1 \\
$|\chi\rangle_{1234}^{(16)}$ & -1 & +1 & -1 & +1 \\
\hline
\end{tabular}

$$
\left.\pm\left(\begin{array}{l}
|1\rangle \\
|0\rangle
\end{array}\right)_{1} \otimes\left(\begin{array}{l}
|\chi\rangle_{2345}^{(2)} \\
|\chi\rangle_{2345}^{(4)} \\
|\chi\rangle_{2345}^{(6)} \\
|\chi\rangle_{2345}^{(8)} \\
|\chi\rangle_{2345}^{(10)} \\
|\chi\rangle_{2345}^{(12)} \\
|\chi\rangle_{2345}^{(14)} \\
|\chi\rangle_{2345}^{(16)}
\end{array}\right) \otimes\left(\begin{array}{c}
|1\rangle \\
|0\rangle
\end{array}\right)_{6}\right]
$$

The $2 N$-particle generalization of the above which contains a set of maximally entangled states can be written down immediately as

$$
|\chi\rangle_{12 \ldots(2 N-1) 2 N}^{(1)-\left(2^{2 N}\right)}=\frac{1}{\sqrt{2}}\left(\left(\begin{array}{c}
|0\rangle \\
|1\rangle
\end{array}\right)_{1} \otimes\left(\begin{array}{c}
|\chi\rangle_{23 \ldots(2 N-1)}^{(1)} \\
|\chi\rangle_{23 \ldots(2 N-1)}^{(3)} \ldots \\
\vdots \\
|\chi\rangle_{23 \ldots(2 N-1)}^{\left(2^{2 N-2}-3\right)} \\
|\chi\rangle_{23 \ldots(2 N-1)}^{\left(2^{2 N-2}-1\right)}
\end{array}\right) \otimes\left(\begin{array}{c}
|0\rangle \\
|1\rangle
\end{array}\right)_{2 N}\right.
$$

$$
\pm\left(\begin{array}{c}
|1\rangle \\
|0\rangle
\end{array}\right)_{1} \otimes\left(\begin{array}{c}
|\chi\rangle_{23 \ldots(2 N-1)}^{(2)} \\
|\chi\rangle_{23 \ldots(2 N-1)}^{(4)} \\
\vdots \\
|\chi\rangle_{23 \ldots(2 N-1)}^{\left(2^{2 N-2}-2\right)} \\
|\chi\rangle_{23 \ldots(2 N-1)}^{\left(2^{2 N-2}\right)}
\end{array}\right) \otimes\left(\begin{array}{c}
|1\rangle \\
|0\rangle
\end{array}\right) \text { 2N }
$$

These states can be used as quantum resources for information transfer between two or more than two parties.

\section{Teleportation using density matrices}

All states, either pure or mixed, require the use of density operator approach for a consistent and correct description of dynamical aspects. In the present instance, density matrix approach is best suited for following processes which are based on the quantum part of entanglement. When the system is not in an eigenstate of the property being measured, the process of measurement introduces a statistical character to the outcome even though the dynamics is given by a deterministic equation. Superimposed on this is a second statistics which is necessary to be consid- 
ered when the state of the system cannot be described by a wave function except to within a probability. Alternately, the system evolves in such a way that different interacting components of the system have different wave functions. Such a situation brings in an additional statistical character to the description/ measurement of the properties of the system.

Following Fano, ${ }^{29}$ a single particle (spin-1/2 or photon) density operator is defined by a $2 \times 2$ Hermitian matrix which is given by a linear combination of Pauli matrices as

$$
\begin{aligned}
\rho & =\frac{1}{2}\left(I+P_{x} \sigma_{x}+P_{y} \sigma_{y}+P_{z} \sigma_{z}\right) \\
& =\frac{1}{2}\left(\begin{array}{cc}
1+P_{z} & P_{x}-i P_{y} \\
P_{x}+i P_{y} & 1-P_{z}
\end{array}\right),
\end{aligned}
$$

where $P_{x}, P_{y}$ and $P_{z}$ are components of polarization vector $\vec{P}$ associated with the particle. A two spin$1 / 2$ density operator can be defined likewise by taking a linear superposition of all the 16 direct product operators

$$
\rho^{12}=\frac{1}{2}\left(I^{1}+P^{1} \cdot \sigma^{1}\right) \frac{1}{2}\left(I^{2}+P^{2} \cdot \sigma^{2}\right) .
$$

Here $\rho^{12}\left(\rho^{1} \otimes \rho^{2}\right)$ is a disentangled state i.e. there is no quantum correlation existing between the spins. The density operator with correlated orientations is

$$
\rho^{12}=\frac{1}{4}\left[\left(I^{1}+P^{1} \cdot \sigma^{1}\right)\left(I^{2}+P^{2} \cdot \sigma^{2}\right)+\sigma^{1} \cdot \underset{\sim}{C} \cdot \sigma^{2}\right]
$$

where the parameter $\underset{\tilde{r}}{\boldsymbol{C}}$ represents the second rank tensor mentioned earliễr, i.e.

$$
\underset{\approx}{C}=\left(\begin{array}{lll}
C_{x x}^{12} & C_{x y}^{12} & C_{x z}^{12} \\
C_{y x}^{12} & C_{y y}^{12} & C_{y z}^{12} \\
C_{z x}^{12} & C_{z y}^{12} & C_{z z}^{12}
\end{array}\right),
$$

whose components $C_{i j}^{12}(1)$ represent the correlation between the two spins. The two-particle density operator can be extended to a three-particle system by appropriately defining the correlation tensors of higher ranks.

\subsection{Teleportation and statistics of the system}

To discuss the importance of statistical correlations and the polarization vectors associated with the particles we have analysed the original teleportation scheme using a two-particle correlated density matrix given as above. Consistent to the scheme proposed by Bennett et al, the unknown state of particle 1 with Alice is represented by a single particle density operator, as

$$
\rho^{1}=|\psi\rangle_{1}\left\langle\left.\psi\right|_{1}=\left(\begin{array}{ll}
|a|^{2} & a b^{*} \\
a^{*} b & |b|^{2}
\end{array}\right) .\right.
$$

Alice interacts her particle with the pair density operator in which the particles of the pair are shared between her and Bob and projects her particles on to one of the four possible Bell states. The correlations between the pair of particles can be consistently described using the Fano's pair density matrix (11). We carry out this calculation using the corresponding projection operators $A_{12}$ and $S_{12}$, which are antisymmetric projection operator corresponding to spin quantum number $F=0$ and symmetric projection operator corresponding to spin quantum number $F=1$, respectively. $S_{12}$ is further decomposed as

$$
S^{12}=S_{x}^{12}+S_{y}^{12}+S_{z}^{12} .
$$

The projectors are given by

$$
\begin{aligned}
A^{12}=|\psi\rangle_{12}^{-}\left\langle\left.\psi\right|_{12} ^{-}\right. & =\frac{1}{4}\left[I^{1} \cdot I^{2}-\sigma^{1} \cdot \sigma^{2}\right] \\
& =\frac{1}{2}\left(\begin{array}{cccc}
0 & 0 & 0 & 0 \\
0 & 1 & -1 & 0 \\
0 & -1 & 1 & 0 \\
0 & 0 & 0 & 0
\end{array}\right)
\end{aligned}
$$

and

$$
S^{12}=\frac{1}{4}\left[3 I^{1} \cdot I^{2}+\sigma^{1} \cdot \sigma^{2}\right],
$$

such that

$$
S_{x}^{12}=|\phi\rangle_{12}^{-}\left\langle\left.\phi\right|_{12} ^{-}=\frac{1}{4}\left[I^{1} \cdot I^{2}+\sigma^{1} \cdot \sigma^{2}-2 \sigma_{x}^{1} \sigma_{x}^{2}\right]\right.
$$

$$
=\frac{1}{2}\left(\begin{array}{cccc}
1 & 0 & 0 & -1 \\
0 & 0 & 0 & 0 \\
0 & 0 & 0 & 0 \\
-1 & 0 & 0 & 1
\end{array}\right),
$$




$$
\begin{aligned}
S_{y}^{12}=|\phi\rangle_{12}^{+}\left\langle\left.\phi\right|_{12} ^{+}\right. & =\frac{1}{4}\left[I^{1} \cdot I^{2}+\sigma^{1} \cdot \sigma^{2}-2 \sigma_{y}^{1} \sigma_{y}^{2}\right] \\
& =\frac{1}{2}\left(\begin{array}{cccc}
1 & 0 & 0 & 1 \\
0 & 0 & 0 & 0 \\
0 & 0 & 0 & 0 \\
1 & 0 & 0 & 1
\end{array}\right)
\end{aligned}
$$

and

$$
\begin{aligned}
S_{z}^{12}=|\psi\rangle_{12}^{+}\left\langle\left.\psi\right|_{12} ^{+}\right. & =\frac{1}{4}\left[I^{1} \cdot I^{2}+\sigma^{1} \cdot \sigma^{2}-2 \sigma_{z}^{1} \sigma_{z}^{2}\right] \\
& =\frac{1}{2}\left(\begin{array}{llll}
0 & 0 & 0 & 0 \\
0 & 1 & 1 & 0 \\
0 & 1 & 1 & 0 \\
0 & 0 & 0 & 0
\end{array}\right) .
\end{aligned}
$$

The corresponding projected components of the pair density matrix are

$$
\left.\rho_{12}^{|\psi\rangle}\right\rangle_{12}^{-}=A^{12} \rho^{12} A^{12}=\frac{\left(1-P^{1} \cdot P^{2}\right)}{4} \cdot \frac{1}{2}\left(\begin{array}{cccc}
0 & 0 & 0 & 0 \\
0 & 1 & -1 & 0 \\
0 & -1 & 1 & 0 \\
0 & 0 & 0 & 0
\end{array}\right),
$$

$$
\rho_{12}^{|\phi\rangle_{12}^{-}}=S_{x}^{12} \rho^{12} S_{x}^{12}=\frac{\left(1+P^{1} \cdot P^{2}-2 P_{x}^{1} P_{x}^{2}-2 C_{x x}^{12}\right)}{4} .
$$

$$
\begin{aligned}
\frac{1}{2}\left(\begin{array}{cccc}
1 & 0 & 0 & -1 \\
0 & 0 & 0 & 0 \\
0 & 0 & 0 & 0 \\
-1 & 0 & 0 & 1
\end{array}\right), \\
\rho_{12}^{|\phi\rangle_{12}^{+}}=S_{y}^{12} \rho^{12} S_{y}^{12}=\frac{\left(1+P^{1} \cdot P^{2}-2 P_{y}^{1} P_{y}^{2}-2 C_{y y}^{12}\right)}{4} .
\end{aligned}
$$$$
\frac{1}{2}\left(\begin{array}{llll}
1 & 0 & 0 & 1 \\
0 & 0 & 0 & 0 \\
0 & 0 & 0 & 0 \\
1 & 0 & 0 & 1
\end{array}\right)
$$

and

$$
\begin{aligned}
& \rho_{12}^{|\psi\rangle_{12}^{+}}=S_{z}^{12} \rho^{12} S_{z}^{12}=\frac{\left(1+P^{1} \cdot P^{2}-2 P_{z}^{1} P_{z}^{2}-2 C_{z z}^{12}\right)}{4} . \\
& \frac{1}{2}\left(\begin{array}{llll}
0 & 0 & 0 & 0 \\
0 & 1 & 1 & 0 \\
0 & 1 & 1 & 0 \\
0 & 0 & 0 & 0
\end{array}\right)
\end{aligned}
$$

such that

$$
\operatorname{Tr} \rho^{12}=\operatorname{Tr}\left[\rho_{12}^{|\psi\rangle_{12}^{-}}+\rho_{12}^{|\phi\rangle_{12}^{-}}+\rho_{12}^{\mid \phi \phi_{12}^{+}}+\rho_{12}^{|\psi\rangle_{12}^{+}}\right],
$$

but

$$
\rho^{12} \neq \rho_{12}^{|\psi\rangle_{12}^{-}}+\rho_{12}^{|\phi\rangle_{12}^{-}}+\rho_{12}^{|\phi\rangle_{12}^{+}}+\rho_{12}^{\mid \psi \psi_{12}^{+}} .
$$

The above equations include correlation that exists between the particles in any entangled pair density matrix. We propose the study of teleportation with correlated and entangled pair density matrices. In accordance with the scheme proposed, the threeparticle density matrix with Alice that leads to her successful measurement of particles 1 and 2 in a pure state (Bell states) is

$$
\rho^{123}=\rho^{1} \otimes \rho_{23}^{|\psi\rangle^{-}} .
$$

The process of Alice's projection measurements for the pair (12) is identical, in principle, to the process of the formation of the correlated pair density matrix formed as entangled pair (23) used as a carrier for teleportation. We have introduced the correlated density matrix (23) earlier as an alternative to the impossibility of experimental preparation of an absolutely pure entangled state between particles (2) and (3) and its subsequent redirection towards Alice and Bob. It is therefore consistent to assume that Alice will not be able to make an absolutely pure entangled pair (12) for $\rho^{123}$, but her measurements would be one of the four possible projections for pair (12) analogous to those for pair (23) given by the (18)-(21). These imply that $\rho^{123}$ when Alice measures her pair of entangled particles (12) must become

$$
\rho^{123} \approx \frac{\left(1-P^{2} \cdot P^{3}\right)}{4} \cdot \frac{1}{4}\left[\begin{array}{l}
|\psi\rangle_{12}^{-}\left\langle\left.\psi\right|_{12} ^{-}\left(1-P^{1} \cdot P^{2}\right) \rho^{3}\right. \\
+|\phi\rangle_{12}^{-}\left\langle\phi | _ { 1 2 } ^ { - } \left( 1+P^{1} \cdot P^{2}\right.\right. \\
\left.-2 P_{x}^{1} P_{x}^{2}-2 C_{x x}^{12}\right) \sigma_{x}^{3} \cdot \rho^{3} \cdot \sigma_{x}^{3} \\
+|\phi\rangle_{12}^{+}\left\langle\phi | _ { 1 2 } ^ { + } \left( 1+P^{1} \cdot P^{2}\right.\right. \\
\left.-2 P_{y}^{1} P_{y}^{2}-2 C_{y y}^{12}\right) \sigma_{y}^{3} \cdot \rho^{3} \cdot \sigma_{y}^{3} \\
+|\psi\rangle_{12}^{+}\left\langle\psi | _ { 1 2 } ^ { + } \left( 1+P^{1} \cdot P^{2}\right.\right. \\
\left.-2 P_{z}^{1} P_{z}^{2}-2 C_{z z}^{12}\right) \sigma_{z}^{3} \cdot \rho^{3} \cdot \sigma_{z}^{3}
\end{array}\right],
$$

where

$$
\rho^{3}=\left(\begin{array}{ll}
|a|^{2} & a b^{*} \\
a^{*} b & |b|^{2}
\end{array}\right)
$$


and is equivalent to the state $\rho^{1}$ which Alice wanted to communicate with Bob. Similarly, if the entangled pair shared between Alice and Bob is $|\phi\rangle_{23}^{+}$instead of $|\psi\rangle_{23}^{-}$then the final three-particle density matrix just before Alice's measurement is

$$
\left.\begin{array}{c}
\rho^{123} \approx \frac{\left(1+P^{2} \cdot P^{3}-2 P_{y}^{2} P_{y}^{3}-2 C_{y y}^{23}\right)}{4} . \\
\frac{1}{4}\left[\begin{array}{l}
|\phi\rangle_{12}^{+}\left\langle\left.\phi\right|_{12} ^{+}\right. \\
\left(1+P^{1} \cdot P^{2}-2 P_{y}^{1} P_{y}^{2}-2 C_{y y}^{12}\right) \rho^{3} \\
+|\psi\rangle_{12}^{-}\left\langle\left.\psi\right|_{12} ^{-}\right. \\
\left(1-P^{1} \cdot P^{2}\right) \sigma_{x}^{3} \cdot\left(\sigma_{z}^{3} \cdot\left(\begin{array}{ll}
|b|^{2} & -a^{*} b \\
-a b^{*} & |a|^{2}
\end{array}\right) \cdot \sigma_{z}^{3}\right.
\end{array}\right) \cdot \sigma_{x}^{3} \\
\left(1+P^{1} \cdot P^{2}-\left.2\right|_{12} ^{-}\right. \\
+|\psi\rangle_{12}^{+}\left\langle\left.\psi\right|_{12} ^{+} P_{x}^{2}-2 C_{x x}^{12}\right) \sigma_{z}^{3}\left(\begin{array}{ll}
|a|^{2} & -a b^{*} \\
-a^{*} b & |b|^{2}
\end{array}\right) \cdot \sigma_{z}^{3} \\
\left(1+P^{1} \cdot P^{2}-2 P_{z}^{1} P_{z}^{2}-2 C_{z z}^{12}\right) \sigma_{x}^{3} \cdot\left(\begin{array}{cc}
|b|^{2} & a b^{*} \\
a^{*} b & |a|^{2}
\end{array}\right) \cdot \sigma_{x}^{3}
\end{array}\right] .
$$

The above analysis preserves only the trace of threeparticle density matrix before and after the measurements. The probability of teleportation without classical communication obviously depends on the statistical correlations and product of polarization vectors existing between the three particles as given by (23) and (24). This opens up the prospect of studying the teleportation of single as well as multiparticle systems by considering the statistics of the system fundamentally and not merely as the outcome for coincidence measurements. The dependence of the probability of teleporting a particle on statistical correlation and polarization vectors also provides an opportunity to study the fidelity associated with the process.

To discuss the teleportation of a single particle through three-particle entangled state we emphasize on Karlsson's proposal of controlled teleportation where a single particle is teleported using threeparticle GHZ state as a quantum channel. ${ }^{30}$ The GHZ state

$$
|\psi\rangle_{234}^{\mathrm{GHZ}}=\frac{1}{\sqrt{2}}\left[|000\rangle_{234}+|111\rangle_{234}\right]
$$

is shared by Alice (2), Charlie (3) and Bob (4). Alice interacts her unknown particle with the GHZ state and measures her two particles in Bell basis and sends her measurement outcome to both Charlie and Bob through classical channel. Charlie assists her by measuring his particles in von Neumann basis and sends his measurement results to Bob. Being consistent with Karlsson's article, if Alice's and Charlie's measurement outcomes are

$$
|\phi\rangle_{12}^{ \pm} \text {and }\left|x_{1}\right\rangle_{3}=\frac{|0\rangle_{3} \pm|1\rangle_{3}}{\sqrt{2}}
$$

respectively, then the process results in a 'successful teleportation' where no unitary transformation is needed on Bob's side. The part of expression for four-particle density matrix depending on statistical correlations is

$$
\rho^{1234} \approx \frac{G^{234}}{8}\left[\begin{array}{l}
|\phi\rangle_{12}^{+}\left\langle\left.\phi\right|_{12} ^{+} \mid x_{1}\right\rangle_{3}\left\langlex _ { 1 } | _ { 3 } \left( 1+P^{1} \cdot P^{2}\right.\right. \\
\left.-2 P_{y}^{1} P_{y}^{2}-2 C_{y y}^{12}\right) \rho^{4}+|\phi\rangle_{12}^{-}\left\langle\left.\phi\right|_{12} ^{-}\right. \\
\left|x_{2}\right\rangle_{3}\left\langle\left. x_{2}\right|_{3}\left(1+P^{1} \cdot P^{2}-2 P_{x}^{1} P_{x}^{2}-2 C_{x x}^{12}\right) \rho^{4}\right.
\end{array}\right]
$$

where

$$
G^{234}=\frac{1}{8}\left[\begin{array}{l}
1+\left(P_{z}^{2} P_{z}^{3}+P_{z}^{3} P_{z}^{4}+P_{z}^{2} P_{z}^{4}\right) \\
+\left(P_{x}^{2} P_{x}^{3} P_{x}^{4}-P_{x}^{2} P_{y}^{3} P_{y}^{4}\right. \\
\left.-P_{y}^{2} P_{x}^{3} P_{y}^{4}-P_{y}^{2} P_{y}^{3} P_{x}^{4}\right) \\
+\left(C_{x x x}^{234}-C_{y y x}^{234}-C_{y x y}^{234}-C_{x y y}^{234}\right)
\end{array}\right] .
$$

The same scheme can be accomplished by using a three-particle $\mathrm{W}$ state $|\psi\rangle_{234}^{W}$ instead of GHZ state, ${ }^{31}$ however, in this case the teleportation is probabilistic and not $100 \%$ as in the case of GHZ state. The measurements that lead towards direct teleportation are $|\psi\rangle_{12}^{+}$at Alice's location and $|0\rangle_{3}$ at Charlie's location as Charlie measures his particles in the basis $|0\rangle_{3}$ and $|1\rangle_{3}$. The density matrix for four particles which results in Alice's successful measurement of an entangled pair is

$$
\rho^{1234} \approx \frac{W^{234}}{6}\left[\begin{array}{l}
|\psi\rangle_{12}^{+}\left\langle\left.\psi\right|_{12} ^{+} \mid 0\right\rangle_{3}\left\langle0 | _ { 3 } \left( 1+P^{1} \cdot P^{2}\right.\right. \\
\left.-2 P_{z}^{1} P_{z}^{2}-2 C_{z z}^{12}\right) \rho^{4}
\end{array}\right],
$$

where 


$$
W^{234}=\frac{1}{24}\left[\begin{array}{l}
3+2\left(P^{2} \cdot P^{3}+P^{3} \cdot P^{4}+P^{2} \cdot P^{4}\right) \\
-3\left(P_{z}^{2} P_{z}^{3}+P_{z}^{3} P_{z}^{4}+P_{z}^{2} P_{z}^{4}\right) \\
+2\left(P_{z}^{2} P_{x}^{3} P_{x}^{4}+P_{z}^{2} P_{y}^{3} P_{y}^{4}\right. \\
+P_{x}^{2} P_{z}^{3} P_{x}^{4}+P_{y}^{2} P_{z}^{3} P_{y}^{4}+P_{x}^{2} P_{x}^{3} P_{z}^{4} \\
\left.+P_{y}^{2} P_{y}^{3} P_{z}^{4}\right)-3 P_{z}^{2} P_{z}^{3} P_{z}^{4} \\
+2\left(C_{x x z}^{234}+C_{y y z}^{234}+C_{x z x}^{234}+C_{y z y}^{234}\right. \\
\left.+C_{z x x}^{234}+C_{z y y}^{234}\right)-3 C_{z z z}^{234}
\end{array}\right] .
$$

In both the above schemes the total probability of teleportation depends on statistical correlation between the four-particles. Thus our discussion of teleportation of single particle through two and threeparticle entangled states based on statistical correlations shows a path to interpret the whole process in terms of statistical parameters. It can be simply extended for the study of multi-particle teleportation using three and more than three-particle entangled states and also to decoherence through relaxation of coherent states with surroundings. The correlation coefficients would then become time-dependent.

\section{Conclusion}

We have discussed a criterion to assess the degree of entanglement and have developed it through a stepwise process, using standard examples, which overcomes the difficulties in the generalization of an entanglement criterion for multi-particle systems. A simple way to generalize four-particle cluster states to a $2 N$-particle entangled system is discussed. Density matrix approach is used to analyse the statistics of teleportation process and a relation between probability of teleportation with statistical parameters is established.

\section{Acknowledgment}

AK is grateful to Indian Institute of Technology (IIT) Madras for a graduate Fellowship. MSK would like to acknowledge IIT Madras for research funds.

\section{References}

1. Bennett C H, Brassard G, Crepeau C, Jozsa R, Peres A and Wootters W K 1993 Phys. Rev. Lett. 701895

2. Bennett C H and Wiesner S J 1992 Phys. Rev. Lett. 692881

3. Zukowski M, Zeilinger A, Horne M A and Ekert A 1993 Phys. Rev. Lett. 714287

4. Schumacker B 1995 Phys. Rev. A51 2738

5. Einstein A, Podolsky B and Rosen N 1935 Phys. Rev. 47777

6. Bohm D and Aharanov Y 1957 Phys. Rev. 1081070

7. Bennett $\mathrm{C} \mathrm{H}$, Bernstein $\mathrm{H}$ J, Popescu $\mathrm{S}$ and Schumacher B 1996 Phys. Rev. A53 2046

8. Hill S and Wootters W K 1997 Phys. Rev. Lett. 785022

9. Wootters W K 1998 Phys. Rev. Lett. 802245

10. Coffman V, Kundu J and Wootters W K 2000 Phys. Rev. A61 052306

11. Vedral V, Plenio M B, Rippin M A and Knight P L 1997 Phys. Rev. Lett. 782275

12. Bouwmeester D, Pan J W, Daniell M, Weinfurter H and Zeilinger A 1999 Phys. Rev. Lett. 821345

13. Pan J W, Daniell M, Gasparoni S, Weihs G and Zeilinger A 2001 Phys. Rev. Lett. 864435

14. Weinfurter H and Zukowski M 2001 Phys. Rev. A64 010102

15. Zhao Z, Chen Y A, Zhang A N, Briegel H J and Pan J W 2004 Nature 43054

16. Walther P, Resch K J, Rudolph T, Schenck E, Weinfurter $\mathrm{H}$, Vedral V, Aspelmeyer $\mathrm{M}$ and Zeilinger A 2005 Nature 434169

17. Schlienz J and Mahler G 1995 Phys. Rev. A52 4396

18. Altafini C 2004 Phys. Rev. A69 012311

19. Huang K 1987 Statistical mechanics (New York: John Wiley)

20. Fano U 1983 Rev. Mod. Phys. 55855

21. Osborn T A 1977 Phys. Rev. A16 334

22. Watson K M 1956 Phys. Rev. 103489

23. Audenaert M R and Plenio M B 2006 New. J. Phys. 8 266

24. Greenberg D M, Horne M and Zeilinger A 1989 Bell's theorem, quantum theory, and conceptions of the universe (ed.) M Kafatos (Kluwer: Dordrecht)

25. Dur W, Vidal G and Cirac J I 2000 Phys. Rev. A62 062314

26. Rigolin G 2005 Phys. Rev. A71 032303

27. Yeo Y and Chua W K 2006 Phys. Rev. Lett. 96 060502

28. Briegel H J and Raussendorf R 2001 Phys. Rev. Lett. 86910

29. Fano U 1957 Rev. Mod. Phys. 2974

30. Karlsson A and Bourennane M 1998 Phys. Rev. A58 4394

31. Shi B S and Tomita A 2002 Phys. Lett. A296 161 